\title{
On multi-stage production/inventory systems under stochastic demand
}

\author{
Henk Zijm ${ }^{\mathrm{a}, *}$, Geert-Jan Van Houtum ${ }^{\mathrm{b}}$ \\ "University of Twente, Department of Mechanical Engineering, P.O. Box 217, 7500 AE Enschede, The Netherlands \\ ${ }^{\mathrm{h}}$ Eindhoven University of Technology, Department of Mathematics and Computing Science, P.O. Box 513,5600 MD Eindhoven. \\ The Netherlands
}

\begin{abstract}
This paper was presented at the 1992 Conference of the International Society of Inventory Research in Budapest, as a tribute to professor Andrew C. Clark for his inspiring work on multi-echelon inventory models both in theory and practice. It reviews and extends the work of the authors on periodic review serial and convergent multi-echelon systems under stochastic stationary demand. In particular, we highlight the structure of echelon cost functions which play a central role in the derivation of the decomposition results and the optimality of base stock policies. The resulting optimal base stock policy is then compared with an MRP system in terms of cost effectiveness, given a predefined target customer service level. Another extension concerns an at first glance rather different problem; it is shown that the problem of setting safety leadtimes in a multi-stage production-to-order system with stochastic lead times leads to similar decomposition structures as those derived for multi-stage inventory systems. Finally, a discussion on possible extensions to capacitated models, models with uncertainty in both demand and production lead time as well as models with an aborescent structure concludes the paper.
\end{abstract}

\section{Introduction}

This paper reviews and extends the work of the authors on the analysis of periodic review multistage production/inventory system under stationary stochastic demand. It was presented in a special session of the ISIR Conference in Budapest, August 1992, to honor the path-breaking work of Professor A.C. Clark on multi-echelon inventory models, starting at the Rand Corporation in the late $1950 \mathrm{~s}$ and later applied when, e.g., developing multi-

*Corresponding author. indenture, multi-echelon inventory systems for the American Navy.

The publication of Forrester's study on the cyclical variation of stocks in large production-distribution chains [1] marked the start of a period of increasing interest in multi-stage inventory systems. The first rigorous analysis of serial multi-stage inventory systems under random demand was given by Clark and Scarf [2], in a discounted cost dynamic programming framework and based on Clark's observations of the fundamental role played by echelon stocks $[3,4]$. Since then, the literature has expanded rapidly, considering not only serial systems but in particular also systems 
with an aborescense (divergent or distribution) structure and, to a minor extent, systems with an inverse aborescense (convergent or assembly) structure. Clark and Scarf [5] extended their previous work on serial systems with approximate models to handle fixed costs at the upstream stages. Langenhoff and Zijm [6] present an average cost unifying framework which can handle all three structures mentioned above. Cohen and Lee [7] present a completely different approach, based on queueing approximations, to deal with capacitated multi-echelon serial systems. Attention to convergent assembly structures was further given by Schmidt and Nahmias [8], Rosling [9] and Van Houtum and Zijm [10]; in particular the latter two papers establish equivalence properties with serial systems (also discussed in [6]. Most attention has been paid to systems with an aborescense (divergent) structure, more specific to the two-stage single-depot multi-warehouse system under stationary stochastic demand (a few references are [11-22]). Finally, many authors consider lot-sizing problems in deterministic multi-stage production systems. For further details, the reader is referred to the books of Schwarz [23] and Axsäter et al. [24].

This paper discusses periodic review stochastic multi-stage systems with both a serial and an assembly structure. Parts of the unifying framework to be discussed are published elsewhere $[6,10]$. In Section 2, we provide some intuition for the structure of echelon cost functions, after which a brief summary of results obtained so far is given. New results on comparisons with MRP-controlled systems are presented in Section 3 and equivalence relations with safety lead time multi-stage models are discussed in Section 4. Finally, we explore the possibilities to extend the framework to capacitated systems (Section 5) as well as to a series of related but more general interesting models (Section 6).

\section{Multi-stage serial systems and assembly systems: A review}

Consider first a two-stage inventory system where between the two stages and after the most downstream stage material can be stocked in installations. Demand originates at the lowest (down- stream) installation only. The stages (followed by their installations) are numbered such that material flows through stage 2 and stage 1 subsequently. A fixed lead time $l_{2}$ is required to transfer material to installation 2 while the transfer from installation 2 to installation 1 takes a fixed lead time $l_{1}$. These transfers may represent a material supply and production stage, respectively. $F_{l}$ denotes the distribution function of the $l$-period cumulative demand $u_{i}$. If $l=1$, we suppress the index. At the end of each period (of unit size), material may be ordered at each installation (from the next higher installation or from the outside supplier). We assume that there is no material shortage at the outside supplier, hence every order of installation 2 will indeed be delivered $l_{2}$ periods later. Orders from installation 1 , however, may be subject to an additional delay (apart from the lead time $l_{1}$ ) due to a material shortage at installation 2 . We assume that any excess demand is backlogged. Furthermore, a holding $\operatorname{cost} h_{2}$ is incurred at installation 2 and in transfer to installation 1 whereas in the latter installation a holding cost $h_{2}+h_{1}$ is assumed (higher because of value added). Finally, a penalty cost $p$ holds in case of shortage at installation 1 (unfilled demand). All these costs are linear, both in time and quantity. No fixed ordering costs are assumed. Furthermore, because we present an average cost analysis and all excess demand is backlogged, it is easy to verify that linear variable ordering costs do not influence any control policy and therefore can be omitted. All costs are incurred at the end of a period.

The concept of echelon stock for a given installation was introduced by Clark [3] to denote all stock at that installation plus in transit to or on hand at any installation downstream minus eventual backlogs at the most downstream installation. The chain under consideration is called the echelon. An echelon stock may be negative, indicating that the backlogs are larger than the total inventory in that echelon. Echelons are numbered according to the highest installation in that echelon. Finally, we define the echelon inventory position of an installation as its echelon stock plus all material in transfer to that installation.

Clark and Scarf [2] assumed the existence of convex cost functions which depend on echelon 
stocks only. For the linear (inventory and penalty) cost structure defined earlier, we first have to develop a natural definition for the corresponding echelon cost functions. A careful analysis of possible situations easily leads to these natural expressions. Let $v_{n}$ be the stock of echelon $n(n=1,2)$ at the end of a period $\left(v_{2} \geqslant v_{1}\right)$. Then we have costs

$$
\begin{aligned}
& h_{2}\left(v_{2}-v_{1}\right)+\left(h_{1}+h_{2}\right) v_{1}=\left(h_{1}+h_{2}\right) v_{1}-h_{2} v_{1} \\
& +h_{2} v_{2} \\
& \text { if } v_{1}>0, \quad v_{2}>0, \\
& h_{2}\left(v_{2}-v_{1}\right)+p\left(-v_{1}\right)=p\left(-v_{1}\right)-h_{2} v_{1}+h_{2} v_{2} \\
& \text { if } v_{1} \leqslant 0, \quad v_{2}>0 \\
& h_{2}\left(v_{2}-v_{1}\right)+p\left(-v_{1}\right)=p\left(-v_{1}\right)-h_{2} v_{1}+h_{2} v_{2} \\
& \text { if } v_{1} \leqslant 0, \quad v_{2} \leqslant 0 .
\end{aligned}
$$

It therefore seems natural to attribute costs $h_{2} v_{2}$ to the echelon stock $v_{2}$, independent of its sign, whereas to $v_{1}$ we attribute either $\left(h_{1}+h_{2}\right) v_{1}-h_{2} v_{1}$ (if $v_{1}>0$ ) or $p\left(-v_{1}\right)-h_{2} v_{1}$ (if $v_{1} \leqslant 0$ ). Note that the second term in the costs of $v_{1}$ is again independent of its sign. As a result we find that, if at the beginning of a period the echelon stock of installation $n$ is increased to $x_{n}$ for $n=1,2$, then the expected costs incurred at the end of a period are equal to $L_{1}\left(x_{1}\right)+L_{2}\left(x_{2}\right)$, where

$$
\begin{aligned}
L_{1}\left(x_{1}\right)= & \left(h_{1}+h_{2}\right) \int_{0}^{x_{1}}\left(x_{1}-u\right) \mathrm{d} F(u)+p \int_{x_{1}}^{\infty}\left(u-x_{1}\right) \\
& \times \mathrm{d} F(u)-h_{2} \int_{0}^{\infty}\left(x_{1}-u\right) \mathrm{d} F(u) \\
= & h_{1} \int_{0}^{\infty}\left(x_{1}-u\right) \mathrm{d} F(u) \\
& +\left(p+h_{1}+h_{2}\right) \int_{x_{1}}^{\infty}\left(u-x_{1}\right) \mathrm{d} F(u)
\end{aligned}
$$

for all $x_{1}$,
$L_{2}\left(x_{2}\right)=h_{2} \int_{0}^{\infty}\left(x_{2}-u\right) \mathrm{d} F(u) \quad$ for all $x_{2}$.

This defines the echelon cost functions. If now, at the beginning of period $t$, the echelon inventory position of installation 2 is increased to $y_{2}$ and, at the beginning of period $t+l_{2}$, the echelon inventory position of installation 1 to $y_{1}$ or as far as possible if $y_{1}$ cannot be reached, then it is not hard to verify that the expected costs at the end of period $t+l_{2}+l_{1}$ are equal to

$$
\begin{aligned}
D_{2}\left(y_{1}, y_{2}\right)= & D_{1}\left(y_{1}\right)+\int_{0}^{\infty} L_{2}\left(y_{2}-u_{l_{2}}\right) \mathrm{d} F_{l_{2}}\left(u_{l_{2}}\right) \\
& +\int_{y_{2}-y_{1}}^{\infty}\left[D_{1}\left(y_{2}-u_{l_{2}}\right)\right. \\
& \left.-D_{1}\left(y_{1}\right)\right] \mathrm{d} F_{l_{2}}\left(u_{l_{2}}\right),
\end{aligned}
$$

where

$D_{1}\left(y_{1}\right)=\int_{0}^{\infty} L_{1}\left(y_{1}-u_{l_{1}}\right) \mathrm{d} F_{l_{1}}\left(u_{l_{1}}\right)$.

Langenhoff and Zijm [6] show that the optimal average cost policy is characterized by two parameters $\left(S_{1}, S_{2}\right)$, where $S_{1}$ minimizes $D_{1}\left(y_{1}\right)$ and $S_{2}$ subsequently minimizes $D_{2}\left(S_{1}, y_{2}\right)$. In other words, the optimal base stock policy $\left(S_{1}, S_{2}\right)$ prescribes that at the beginning of each period the echelon inventory position of echelon $n$ is increased to $S_{n}$, for $n=1$, 2. Analogous results are derived for $N$-stage serial systems, meaning that the $N$-dimensional optimization problem can be solved completely by successively solving a series of recursively defined one-dimensional problems. An extensive numerical analysis of the difficulties stemming from these recursive definitions is presented by Van Houtum and Zijm [10]. The equivalence of serial and assembly systems has been proved by Rosling [9] and Langenhoff and Zijm [6]. Finally, Van Houtum and Zijm [10] show how to extend the analysis to systems in which average holding costs have to be minimized, subject to a target customer service level. 
Table 1

Basic parameter values for the two-stage assembly model

\begin{tabular}{llllll}
\hline$h_{0}=5$ & $h_{1}=1.5$ & $h_{2}=1.5$ & $h_{3}=2$ & $\mu=100$ & $\sigma=70$ \\
$l_{0}-2$ & $l_{1}-1$ & $l_{2}-2$ & $l_{3}-4$ & $\tau-95 \%$ & \\
\hline
\end{tabular}

\section{Comparisons with MRP-controlled systems}

The optimality of a base stock control policy for serial and assembly systems implies that safety stocks, meant to anticipate uncertainty in future demand, are spread over the entire chain. An alternative is to keep all safety stocks at one installation, close to the market. This is the policy advocated in the initial MRP literature (see e.g. [25]) where all uncertainty in a make and assemble to stock environment is covered at the end-item level. Often, the master production schedule (MPS) specifies quantities of end items to be assembled each period while the preceding phases (component manufacturing and subassembly) are driven deterministically by this MPS. More precisely, under conditions similar to those studied in this paper, the MPS of an MRP system should specify for each period how much end items have to be produced to return to an optimal reorder level which covers all demand uncertainty during the full system lead time (plus one period). In fact, this is similar to a singlestage inventory system; the only difference with the classical Newsboy problem is that holding costs are calculated relative to the manufacturing or assembly stage the material is in. Optimal reorder levels for such a, basically single-stage, system are easily determined but it seems worthwhile to investigate the cost differences between such an optimal MRP policy and an overall optimal base stock policy. Note that the two policies coincide if no added value is counted in the system (i.e. $h_{n}=0$ for $n \geqslant 2$ ).

We start with investigating these cost differences for a simple two-stage assembly system with three components and one end item, under a service level constraint. Table 1 lists the basic parameter values (where $\mu$ and $\sigma$ denote the mean and standard deviation of the periodic demand, while $\tau$ is the target customer service level).

In the following tables, a number of cost comparisons between an optimal MRP policy and an overall optimal policy are made as a function of one varying parameter (e.g. $\sigma$ in Table $2, \tau$ in Table 3 ). The order-up-to levels for the optimal base stock policy as well as the (single) order-up-to level for the optimal MRP policy $\left(S_{\mathrm{MRP}}\right)$ are given, together with the inventory holding costs for both policies $\left(C_{\mathrm{BS}}\right.$ and $\left.C_{\mathrm{MRP}}\right)$. CD finally denotes the cost difference between the two policies, expressed both absolutely and as a percentage.

Table 2 illustrates the intuitively obvious result that the differences in cost between an optimal MRP policy and an overall optimal policy increase as a function of demand uncertainty (the standard variation). Table 3 shows that the cost difference is also (weakly) sensitive to the target service level; this sensitivity seems to increase for very high service levels.

Table 2

Comparisons between MRP and base stock policies for varying $\sigma$

\begin{tabular}{rrrrrrrrrr}
\hline$\sigma$ & $S_{0}$ & $S_{1}$ & \multicolumn{1}{c}{$S_{2}$} & \multicolumn{1}{c}{$S_{3}$} & $C_{\mathrm{BS}}$ & $S_{\mathrm{MRP}}$ & $C_{\mathrm{MRP}}$ & CD & CD $(\%)$ \\
\hline 10 & 316.8 & 419.5 & 519.2 & 718.9 & 1174 & 714.2 & 1192 & 18 & 1.5 \\
20 & 346.0 & 455.3 & 557.8 & 763.1 & 1480 & 751.2 & 1562 & 82 & 5.5 \\
30 & 381.3 & 498.4 & 604.4 & 816.4 & 1869 & 7963 & 2013 & 144 & 7.7 \\
40 & 421.7 & 547.4 & 657.2 & 876.6 & 2317 & 847.7 & 2527 & 210 & 9.1 \\
50 & 467.6 & 602.7 & 716.5 & 944.0 & 2827 & 905.1 & 3101 & 274 & 9.7 \\
60 & 516.9 & 661.9 & 779.8 & 1016 & 3376 & 966.6 & 3716 & 340 & 10.1 \\
70 & 573.0 & 728.6 & 850.5 & 1096 & 3995 & 1035 & 4397 & 402 & 10.1 \\
80 & 628.0 & 794.1 & 920.4 & 1175 & 4607 & 1103 & 5078 & 471 \\
90 & 691.3 & 868.1 & 993.3 & 1263 & 5304 & 1179 & 5838 & 534 & 10.2 \\
100 & 772.4 & 961.7 & 1096 & 1371 & 6183 & 1271 & 6755 & 572 & 10.1 \\
\hline
\end{tabular}


Table 3

Comparisons between MRP and base stock policies for varying $\tau$

\begin{tabular}{llllllllll}
\hline$\tau(\%)$ & $S_{0}$ & $S_{1}$ & $S_{2}$ & $S_{3}$ & $C_{\text {BS }}$ & $S_{\text {MRP }}$ & $C_{\text {MRP }}$ & CD & CD (\%) \\
\hline 90 & 522.3 & 667.3 & 781.6 & 1015 & 3384 & 959.8 & 3698 & 314 & 9.3 \\
91 & 530.1 & 676.8 & 792.4 & 1027 & 3478 & 971.5 & 3805 & 327 & 9.4 \\
92 & 538.7 & 687.4 & 804.3 & 1041 & 3583 & 984.5 & 3925 & 342 & 9.5 \\
93 & 548.5 & 699.2 & 817.6 & 1057 & 3701 & 999.0 & 4060 & 359 & 9.7 \\
94 & 559.8 & 712.7 & 832.8 & 1075 & 3836 & 1015 & 4215 & 379 & 9.9 \\
95 & 573.0 & 728.6 & 850.5 & 1096 & 3995 & 1035 & 4397 & 402 & 10.1 \\
96 & 589.1 & 747.7 & 871.8 & 1120 & 4189 & 1058 & 4619 & 430 & 10.3 \\
97 & 609.6 & 771.9 & 898.7 & 1151 & 4435 & 1087 & 4900 & 465 & 10.5 \\
98 & 638.2 & 805.4 & 935.7 & 1194 & 4776 & 1127 & 5291 & 515 & 10.8 \\
99 & 686.3 & 861.0 & 996.7 & 1263 & 5345 & 1193 & 5941 & 596 & 11.2 \\
\hline
\end{tabular}

Table 4

Basic parameter values for the two-stage serial model

$l_{0}=2 l_{1}=4 \quad h_{i}=2 l_{1}$ for $i=0,1 \quad \mu=100 \sigma=70 \quad \tau=95 \%$

In the next tables we investigate the influence of the amount of value added in the manufacturing process, as well as the influence of the lead times. A simple two-stage serial system is used as an example (see Table 4). Since inventory holding costs are usually taken as a percentage of the value of an item at each moment, a large $h_{0}$ value reflects a high added value in the manufacturing process. Intuitively, one would expect a sharp increase in cost difference as $h_{0}$ grows larger and a relatively minor influence of the ratios of the lead times. Tables 5 and 6 confirm these conjectures.

Finally, we investigate the influence of the number of installations in a serial system. Table 7 lists the basic parameters; the results are shown in Table 8 .

In summary, we may conclude that the increase in costs as a result of the absence of buffer stocks in the component and subassembly stores (hence only stocks of end items) strongly depends on the amount of value added during the subsequent stages in the manufacturing process. The influence of the height of the target customer service level is less significant but a high demand variability may have a very serious impact on these cost differences.

Table 5

The influence of the value added in a two-stage serial model

\begin{tabular}{|c|c|c|c|c|c|c|c|c|}
\hline$h_{0}$ & $h_{1}$ & $S_{0}$ & $S_{1}$ & $\mathrm{C}_{\mathrm{BS}}$ & $S_{\text {MRP }}$ & $\mathrm{C}_{\mathrm{MRP}}$ & $C D$ & $\mathrm{CD}(\%)$ \\
\hline 0 & 10 & 1035 & 1035 & 5397 & 1035 & 5397 & 0 & 0.0 \\
\hline 1 & 9 & 703.7 & 1036 & 5186 & 1035 & 5197 & 11 & 0.2 \\
\hline 2 & 8 & 651.7 & 1040 & 4949 & 1035 & 4997 & 48 & 1.0 \\
\hline 3 & 7 & 619.4 & 1045 & 4689 & 1035 & 4797 & 108 & 2.3 \\
\hline 4 & 6 & 595.3 & 1052 & 4409 & 1035 & 4597 & 188 & 4.3 \\
\hline 5 & 5 & 575.4 & 1062 & 4107 & 1035 & 4397 & 290 & 7.1 \\
\hline 6 & 4 & 558.4 & 1075 & 3784 & 1035 & 4197 & 413 & 10.9 \\
\hline 7 & 3 & 543.1 & 1093 & 3435 & 1035 & 3997 & 562 & 16.4 \\
\hline 8 & 2 & 529.0 & 1121 & 3054 & 1035 & 3797 & 743 & 24.3 \\
\hline 9 & 1 & 516.0 & 1171 & 2627 & 1035 & 3597 & 970 & 36.9 \\
\hline 10 & 0 & 503.8 & $\infty$ & 2088 & 1035 & 3397 & 1309 & 62.7 \\
\hline
\end{tabular}


Table 6

The influence of the lead time ratios in a two-stage serial model

\begin{tabular}{rllllllrr}
\hline$l_{0}$ & $l_{1}$ & $S_{0}$ & $S_{1}$ & $C_{\mathrm{BS}}$ & $S_{\text {MRP }}$ & $C_{\text {MRP }}$ & CD & CD (\%) \\
\hline 1 & 5 & 510.4 & 1040 & 4981 & 1035 & 5077 & 96 & 1.9 \\
2 & 4 & 610.7 & 1047 & 5517 & 1035 & 5677 & 160 & 2.9 \\
3 & 3 & 715.8 & 1056 & 5695 & 1035 & 5877 & 182 & 3.2 \\
4 & 2 & 821.7 & 1065 & 5509 & 1035 & 5677 & 168 & 3.0 \\
5 & 1 & 928.0 & 1076 & 4965 & 1035 & 5097 & 112 \\
\hline
\end{tabular}

Table 7

Basic parameters for investigating the influence of $N$

\begin{tabular}{|c|c|c|c|c|c|c|c|c|c|c|c|c|}
\hline$N$ & $h_{0}$ & $h_{1}$ & $h_{2}$ & $h_{3}$ & $h_{4}$ & $h_{5}$ & $l_{0}$ & $l_{1}$ & $l_{2}$ & $l_{3}$ & $l_{4}$ & $l_{5}$ \\
\hline 1 & 5 & 5 & & & & & 2 & 5 & & & & \\
\hline 2 & 5 & 4 & 1 & & & & 2 & 4 & 5 & & & \\
\hline 3 & 5 & 3 & 1 & 1 & & & 2 & 3 & 4 & 5 & & \\
\hline 4 & 5 & 2 & 1 & 1 & 1 & & 2 & 2 & 3 & 4 & 5 & \\
\hline 5 & 5 & 1 & 1 & 1 & 1 & 1 & 2 & 1 & 2 & 3 & 4 & 5 \\
\hline & \multicolumn{3}{|c|}{$\mu=100$} & \multicolumn{4}{|c|}{$\sigma=70$} & \multicolumn{4}{|c|}{$\tau=95 \%$} & \\
\hline
\end{tabular}

\section{A related model: Setting safety lead times in a multi-stage system}

The problem of setting planning lead times in a multi-stage production system in which production is order driven and tardiness results in penalty costs has been investigated by several authors (see e.g. $[26,27]$ ). Here the lead times are assumed to be random variables with a known distribution function. For the single-stage system, the problem of setting a planning or safety lead time leads to a Newsboy formulation. De Kok [28] recognized the striking similarity between the general problem of setting safety lead times in an $N$-stage serial system and the results of the analysis presented in Section 2 for the $N$-stage inventory problem. Below, we show the equivalence of the two problems for the two-stage serial system.

Consider a two-stage serial, order driven, production system with stochastic lead times $\underline{t}_{2}$ (for the upstream or manufacturing stage) and $\underline{t}_{1}$ (for the final assembly stage). Let $F_{n}$ denote the distribution functions of $\underline{t}_{n}$ for $n=1,2$. Suppose, furthermore, that a holding cost $h_{2}$ is incurred for a batch of products (components or subassemblies) in installation 2 or between installations 2 and 1 , while a batch of final products is stored at a rate $h_{2}+h_{1}$ if the batch is finished before its due date. A batch is temporarily stored in installation 2 if it arrives there before the planned starting time of the final stage. If the final stage of the batch is completed after its due date, a linear penalty cost $p$ per unit per period is incurred. A batch is delivered to the customer at its due date or, if not possible, immediately after completion. The problem is to determine the best starting time for each phase, i.e. how to set planning or safety lead times to minimize average holding and penalty costs?

Table 8

The influence of the number of installations $N$

\begin{tabular}{|c|c|c|c|c|c|c|c|c|c|c|c|}
\hline$N$ & $S_{0}$ & $S_{1}$ & $S_{2}$ & $S_{3}$ & $S_{4}$ & $S_{5}$ & $C_{\mathrm{BS}}$ & $S_{\text {MRP }}$ & $C_{\mathrm{MRP}}$ & $\mathrm{CD}$ & $\operatorname{CD}(\%)$ \\
\hline 1 & 577.3 & $119 ?$ & & & & & 4275 & 1162 & 4666 & 391 & 9.1 \\
\hline 2 & 576.3 & 1084 & 1232 & & & & 4225 & 1162 & 4666 & 441 & 10.4 \\
\hline 3 & 575.5 & 975.6 & 1119 & 1237 & & & 4189 & 1162 & 4666 & 477 & 11.4 \\
\hline 4 & 574.9 & 866.0 & 1004 & 1123 & 1239 & & 4167 & 1162 & 4666 & 499 & 12.0 \\
\hline 5 & 574.7 & 754.3 & 883.9 & 1006 & 1124 & 1239 & 4156 & 1162 & 4666 & 510 & 12.3 \\
\hline
\end{tabular}


Define $T_{2}$ as the total planning lead time for the two stages together and $T_{1}$ as the planning lead time for the final (downstream) stage alone. Also, let

$\underline{v}_{2}=T_{2}-\underline{t}_{2}, \quad \underline{v}_{1}=\min \left(\underline{v}_{2}, T_{1}\right)-\underline{t}_{1}$.

Hence, $\underline{v}_{n}$ denotes the time difference between the completion of stage $n$ and the final due date. Then the following cost structure appears:

$$
\begin{array}{ll}
h_{2}\left(\underline{v}_{2}-\underline{v}_{1}\right)+\left(h_{1}+h_{2}\right) \underline{v}_{1} & \\
=\left(h_{1}+h_{2}\right) \underline{v}_{1}-h_{2} \underline{v}_{1}+h_{2} \underline{v}_{2} & \text { if } \underline{v}_{1}>0, \quad \underline{v}_{2}>0, \\
h_{2}\left(\underline{v}_{2}-\underline{v}_{1}\right)+p\left(-\underline{v}_{1}\right) & \\
=p\left(-\underline{v}_{1}\right)-h_{2} \underline{v}_{1}+h_{2} \underline{v}_{2} & \text { if } \underline{v}_{1} \leqslant 0, \quad \underline{v}_{2}>0, \\
h_{2}\left(\underline{v}_{2}-\underline{v}_{1}\right)+p\left(-\underline{v}_{1}\right) & \\
=p\left(-\underline{v}_{1}\right)-h_{2} \underline{v}_{1}+h_{2} \underline{v}_{2} & \text { if } \underline{v}_{1} \leqslant 0, \quad \underline{v}_{2} \leqslant 0 .
\end{array}
$$

The reader may note that this is completely identical to the inventory cost structure in Section 2 which formed the basis of the echelon cost functions. For the safety lead time problem discussed here we find that the optimal planning lead times can be found by minimizing the following function:

$$
\begin{aligned}
C_{2}\left(T_{1}, T_{2}\right)= & C_{1}\left(T_{1}\right)+h_{2} \int_{0}^{\infty}\left(T_{2}-t_{2}\right) \mathrm{d} F_{2}\left(t_{2}\right) \\
& +\int_{T_{2}-T_{1}}^{\infty}\left[C_{1}\left(T_{2}-t_{2}\right)\right. \\
& \left.-C_{1}\left(T_{1}\right)\right] \mathrm{d} F_{2}\left(t_{2}\right),
\end{aligned}
$$

where

$$
\begin{aligned}
C_{1}\left(T_{1}\right)= & \left(h_{1}+h_{2}\right) \int_{0}^{T_{1}}\left(T_{1}-t_{1}\right) \mathrm{d} F_{1}\left(t_{1}\right) \\
& +p \int_{T_{1}}^{\infty}\left(t_{1}-T_{1}\right) \mathrm{d} F_{2}\left(t_{1}\right) \\
& -h_{2} \int_{0}^{\infty}\left(T_{1}-t_{1}\right) \mathrm{d} F_{1}\left(t_{1}\right)
\end{aligned}
$$

$$
\begin{aligned}
& =h_{1} \int_{0}^{\infty}\left(T_{1}-t_{1}\right) \mathrm{d} F_{1}\left(t_{1}\right) \\
& +\left(p+h_{1}+h_{2}\right) \int_{T_{1}}^{\infty}\left(t_{1}-T_{1}\right) \mathrm{d} F_{1}\left(t_{1}\right),
\end{aligned}
$$

where $C_{1}\left(T_{1}\right)$ is a convex function, which is minimized in $T_{1}=L_{1}$, say, and $C_{2}\left(L_{1}, T_{2}\right)$ is a convex function in $T_{2}$. Exactly the same recursive procedure as in Section 2 can therefore be applied to obtain the optimal $\left(T_{1}, T_{2}\right)$-tuple by successively minimizing two one-dimensional functions. Let the optimal values be $L_{1}$ and $L_{2}$; then the optimal planned lead times for the upstream and downstream stages equal $L_{2}-L_{1}$ and $L_{1}$, respectively. The generalization of the above arguments to general $N$-stage serial systems and to systems with an assembly structure are completely straightforward and are therefore left to the reader.

\section{Extensions to capacitated multi-stage systems}

In this section we seek to extend the theoretical framework to systems where transfer at each stage, i.e. between each two installations, is limited by a capacity constraint. Although, in general, we cannot expect that the results derived so far continue to hold in capacitated systems, an important question is whether base-stock policies, based on echelon stocks, are still close to optimal. If the answer to this question is affirmative, numerical schemes taking into account the capacity limitations have to be developed again.

Speck and Van der Wal $[29,30]$ have investigated the first question. We only briefly summarize their results here. Define a serial $N$-stage system as before and assume that transfer between stage $i+1$ and stage $i$ is limited by an amount $c_{i}$ (the production or transfer capacity). Some reflection shows that, without any loss of generality, one may restrict oneself to systems with $c_{i+1} \leqslant c_{i}$. Speck and Van der Wal numerically show that for a two-stage serial system a so-called push-ahead effect may exist. That is, the replenishment decision of the downstream installation may depend on the 
amount of material in transfer to installation 2 (upstream). On the other hand, Speck and Van der Wal provide evidence that in all cases there exist base-stock policies, the calculation of which is based on capacity limitations as well, which are very close to optimal.

In this section, we therefore concentrate on basestock policies again. For the development of numerical procedures we first briefly study the single-stage system. Note that from the results of Federgruen and Zipkin [31] the optimality of an order-up-to level policy easily follows for a singlestage capacitated system, as long as no fixed ordering costs are assumed. Now, consider a two-stage linear system as described in Section 2 with the extra assumption of a limited transfer capacity $c_{2}$ per period to installation 2 while transfers between installation 2 and installation 1 are unconstrained. Let $z_{2}$ denote an order-up-to level for the echelon inventory position of installation 2 (i.e. if, at the beginning of a period, this echelon inventory position is equal to $x_{2}$ then we order $\min \left(z_{2}-x_{2}, c_{2}\right)$ if $x_{2}<z_{2}$ and nothing otherwise). Furthermore, let $y_{2, n}$ be the echelon inventory position of installation 2 just after ordering in period $n$. Then we can write the following traffic equation for $y_{2, n+1}$ and $y_{2, n}$ :

$z_{2}-y_{2, n+1}=\max \left(z_{2}-y_{2, n}+u-c, 0\right)$,

where $u$ denotes the one-period (stochastic) demand. Hence, $z_{2}-y_{2, n}$ satisfies the law of motion of the waiting time (excluding service) of an arriving customer in a $D / G / 1$ queue with interarrival time $c$ and service time $u$. If the mean demand $\mu<c$ (meaning that on average the system can fulfill all demand) then clearly $z_{2}-y_{2, n} \rightarrow z_{2}-y_{2}$ for $n \rightarrow \infty$ with probability one for some random variable $y_{2}$. If we define $w:=z_{2}-y_{2}$ then the probability distribution function $G(w)$ of $w$ satisfies the following Wiener-Hopf equation:

$G(w)=\int_{0}^{w+c} G(w+c-u) \mathrm{d} F(u)$.

Note that $G(w)$ has a discontinuity in 0 (since $G(0)>0)$ while $G(w)$ is differentiable for $w>0$, with derivative $g(w)$, say. De Kok [32] developed a method to quickly determine an approximation of $G(w)$ in terms of a mixture of Erlang distributions which has proven to be extremely accurate. Since the random variable $y_{2}=z_{2}-w$ equals the inventory position just after ordering we now easily find the optimal order-up-to level $S_{2}$ by minimizing the following expression:

$$
\begin{aligned}
E\left(S_{1}, z_{2}\right)= & \int_{0}^{\infty} D_{2}\left(S_{1}, z_{2}-w\right) g(w) \mathrm{d} w \\
& +D_{2}\left(S_{1}, z_{2}\right) G(0)
\end{aligned}
$$

where $S_{1}$ is determined in the first step of the recursive procedure presented in Section 2 for unconstrained systems while also $D_{2}\left(y_{1}, y_{2}\right)$ denotes the two-dimensional cost function given in Section 2. It can be shown, using the results of Federgruen and Zipkin [31], that this procedure still yields an overall optimal policy.

If there exists a capacity constraint $c_{1}$ on the periodic transfer of materials between installation 2 and installation 1 as well, then the term $D_{1}\left(y_{1}\right)$ in the recursive definition of $D_{2}\left(y_{1}, y_{2}\right)$ has to be replaced by a function $E_{1}\left(z_{1}\right)$ which is determined along lines similarly to the analysis given above. The term $D_{1}\left(y_{2}-u_{l_{2}}\right)$ is replaced by a more complicated expression to indicate that there may be two causes for not attaining the order-up-to level $z_{1}$, i.e. a material shortage or a lack of capacity between installation 2 and installation 1 . The analysis of these complications is beyond the scope of this paper; a detailed treatment will be given in a forthcoming publication.

\section{Conclusions and suggestions for further research}

In this paper we have reviewed and extended a theoretical framework for the planning of stochastic multi-stage serial and assembly systems. An equivalence property between these two systems is established. Numerical procedures to actually calculate order-up-to levels have been developed and perform satisfactorily. A translation to a more realistic situation where optimal policies have to be determined subject to a target customer service level constraint follows easily from the cost 
expressions underlying the numerical evaluation. The numerical calculation scheme also allows us to compare the performance of the optimal base-stock policies with an MRP control structure, showing remarkable differences. Furthermore, the problem to determine safety lead times in a stochastic lead time multi-stage system with known demand appears to have a highly similar structure. Finally, we elaborate on possibilities to extend the theory to capacitated multi-stage systems.

Further extensions are possible and are highly recommended for investigation. The extension of the numerical calculation scheme to systems with a divergent (aborescense) structure is currently studied and will be treated in a forthcoming publication (see also [33]). Referring to Section 7, the combination of stochastic demand and stochastic lead times may lead to more realistic models covering unreliability and scheduling effects. Highly important seems to be the extension to systems with a non-stationary demand structure as well as to systems where demand originates not only at the end-item level. In particular, the combination of non-stationary demand (e.g. seasonal patterns) with capacity management issues may lead to a framework which perhaps cannot be treated by exact methods any longer but on the other hand provides us with a tool to understand and manage rather complex practical production/inventory systems.

\section{References}

[1] Forrester, J.W., 1961. Industrial Dynamics. MIT Press, Cambridge, MA.

[2] Clark, A.J. and Scarf, H., 1960. Optimal policies for a multi-echelon inventory problem. Mynt. Sci., 6: 475-490.

[3] Clark, A.J., 1958. A dynamic, single-item, multi-echelon inventory model. RM-2297. 'The Rand Corporation, Santa Monica.

[4] Clark, A.J., 1992. Multi-echelon inventory theory - A retrospective. Presented at the 1992 ISIR Conf., Budapest.

[5] Clark, A.J. and Scarf, H., 1962. Approximate solutions to a simple multi-echelon inventory problem, in: K.J. Arrow et al. (Eds.), Studies in Applied Probability and Management Science. Stanford University Press, Stanford.

[6] Langenhoff, L.J.G. and Zijm, W.H.M., 1990. An analytical theory of multi-echelon production/distribution systems. Statistica Neerlandica, 44(3): 149-174.
[7] Cohen, M.A. and Lee, H.L., 1988. Strategic analysis of integrated production-distribution systems: Models and methods. Oper. Res., 36(2): 216-228.

[8] Schmidt, C.P. and Nahmias, S., 1985. Optimal policy for a two-stage assembly system under random demand. Oper. Res., 33: 1130-1145.

[9] Rosling, K., 1989. Optimal inventory policies for assembly systems under random demands. Oper. Res., 37(4): 565-579.

[10] Van Houtum, G.J. and Zijm, W.H.M., 1991. Computational procedures for stochastic multi-echelon production systems. Int. J. Prod. Econom., 23: 223-237.

[11] Eppen, G. and Schrage, L., 1981. Centralized ordering policies in a multi-warehouse system with lead times and random demand, in: L.B. Schwarz (Ed.), Multi-level Production/Inventory Control Systems: Theory and Practice (Studies in the Management Sciences, Vol. 16), NorthHolland, Amsterdam, pp. 51-67.

[12] Federgruen, A. and Zipkin, P., 1984a. Approximations of dynamic, multilocation production and inventory problems. Mgmt. Sci., 30: 69-84.

[13] Federgruen, A. and Zipkin, P., 1984b. Allocation policies and cost approximations for multilocation inventory systems. Naval Res. Logistic. Quart., 31: 69-84.

[14] Federgruen, A. and Zipkin, P., 1984c. Computational issues in an infinite-horizon, multi-echelon inventory model. Oper. Res., 32: 818-836.

[15] Van Donselaar, K. and Wijngaard, J., 1986. Practical application of the echelon approach in a system with divergent product structure, in: S. Axsäter et al. (Eds.), Multi-stage Production Planning and Inventory Control. Springer, Berlin, pp. 182-196.

[16] Van Donselaar, K. and Wijngaard, J., 1987. Commonality and safety stocks. Eng. Costs Prod. Econom., 12: 197-204.

[17] Axsäter, S., 1990. Simple solution procedures for a class of two-echelon inventory problems. Oper. Res., 38: 64-69.

[18] Jönsson, H. and Silver, E.A., 1987a. Analysis of a two-echelon inventory control system with complete redistribution. Mgmt. Sci., 33: 880-895.

[19] Jönsson, H. and Silver, E.A., 1987b. Stock allocation among a central warehouse and identical regional warehouses in a particular push inventory control system. Int. J. Prod. Res., 25: 191-205.

[20] De Kok, A.G., 1990, Hierarchical production planning for consumer goods. Eur. J. Oper. Res., 45: 55-69.

[21] Inderfurth, K., 1991. Safety stock optimization in multistage inventory systems. Int. J. Prod. Econom., 24: $103-113$.

[22] Inderfurth, K., 1992. Safety stocks in multi-stage divergent inventory systems: A survey. Presented at the 1992 ISIR Conference, Budapest.

[23] Schwarz, L.B. (Ed.), 1981. Multi-level production/inventory control systems: Theory and practice, in: Studies in the Management Sciences, Vol. 16, North-Holland, Amsterdam.

[24] Axsäter, S., Schneeweiss, Ch. Silver, E.A. (Eds.), 1986. Multi-Stage Production Planning and Inventory Control. Springer, Berlin. 
[25] Orlicky, J.A., 1975. Materials Requirements Planning. McGraw-Hill, New York.

[26] Yano, C.A., 1987. Setting planned leadtimes in serial production systems with tardiness costs. Mgmt. Sci., 33(1): 95-106.

[27] Gong, L., De Kok, A.G. and Ding, J., 1992. Optimal leadtimes planning in serial production systems. Dept. of Quantitative Business Analysis, Louisiana State University, Baton Rouge.

[28] De Kok, A.G., 1992, personal communication.

[29] Speck, C.J. and van der Wal, J., 1991a. The capacitated multi-echelon inventory system with serial structure: 1. The 'push ahead'-effect. Memorandum COSOR 91-39, Department of Mathematics and Computing Science, Eindhoven University of Technology, Eindhoven, The Netherlands.

[30] Speck, C.J. and van der Wal, J., 1991b. The capacitated multi-echelon inventory system with serial structure: 2 . An average cost approximation method. Memorandum COSOR 91-40. Department of Mathematics and Computing Science, Eindhoven University of Technology, Eindhoven, The Netherlands.

[31] Federgruen, A. and Zipkin, P., 1986. An inventory model with limited production capacity and uncertain demands. I: The average cost criterion. Math. Oper. Res., 11: 193-207.

[32] De Kok, A.G., 1989. A moment-iteration method for approximating the waiting-time characteristics of the $G I / G / 1$ queue. Probab. Eng. Inform. Sci., 3: 273-287.

[33] Van Houtum, G.J. and Zijm, W.H.M., 1992. Theoretische en Numerieke analyse van multi-echelon voorraadsystemen met distributiestruktuur (in Dutch). Working Paper, Department of Mathematics and Computing Science, Eindhoven University of Technology, Eindhoven, The Netherlands.

[34] Schassberger, R., 1973. Warteschlangen. Springer, Berlin. 